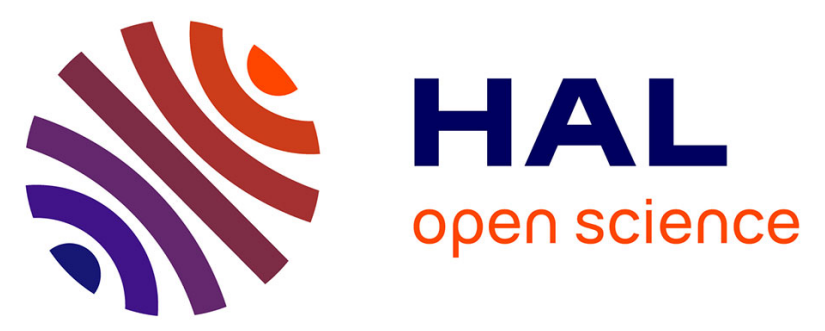

\title{
Comment construire des scénarios prospectifs adaptés à la prise en compte des enjeux de biodiversité tels que les continuités écologiques?
}

\author{
Mathilde Herve, Thierry Tatoni, Cécile H. Albert, Sylvie Vanpeene
}

\section{- To cite this version:}

Mathilde Herve, Thierry Tatoni, Cécile H. Albert, Sylvie Vanpeene. Comment construire des scénarios prospectifs adaptés à la prise en compte des enjeux de biodiversité tels que les continuités écologiques?. Sciences Eaux \& Territoires, 2020, Hors-Série, 71. hal-02997914

\section{HAL Id: hal-02997914 \\ https://hal-amu.archives-ouvertes.fr/hal-02997914}

Submitted on 10 Nov 2020

HAL is a multi-disciplinary open access archive for the deposit and dissemination of scientific research documents, whether they are published or not. The documents may come from teaching and research institutions in France or abroad, or from public or private research centers.
L'archive ouverte pluridisciplinaire HAL, est destinée au dépôt et à la diffusion de documents scientifiques de niveau recherche, publiés ou non, émanant des établissements d'enseignement et de recherche français ou étrangers, des laboratoires publics ou privés. 
Mathilde HERVÉ, Thienry TATONI,

Sylvie VAYPEEN J et Cécile H.ALBERII

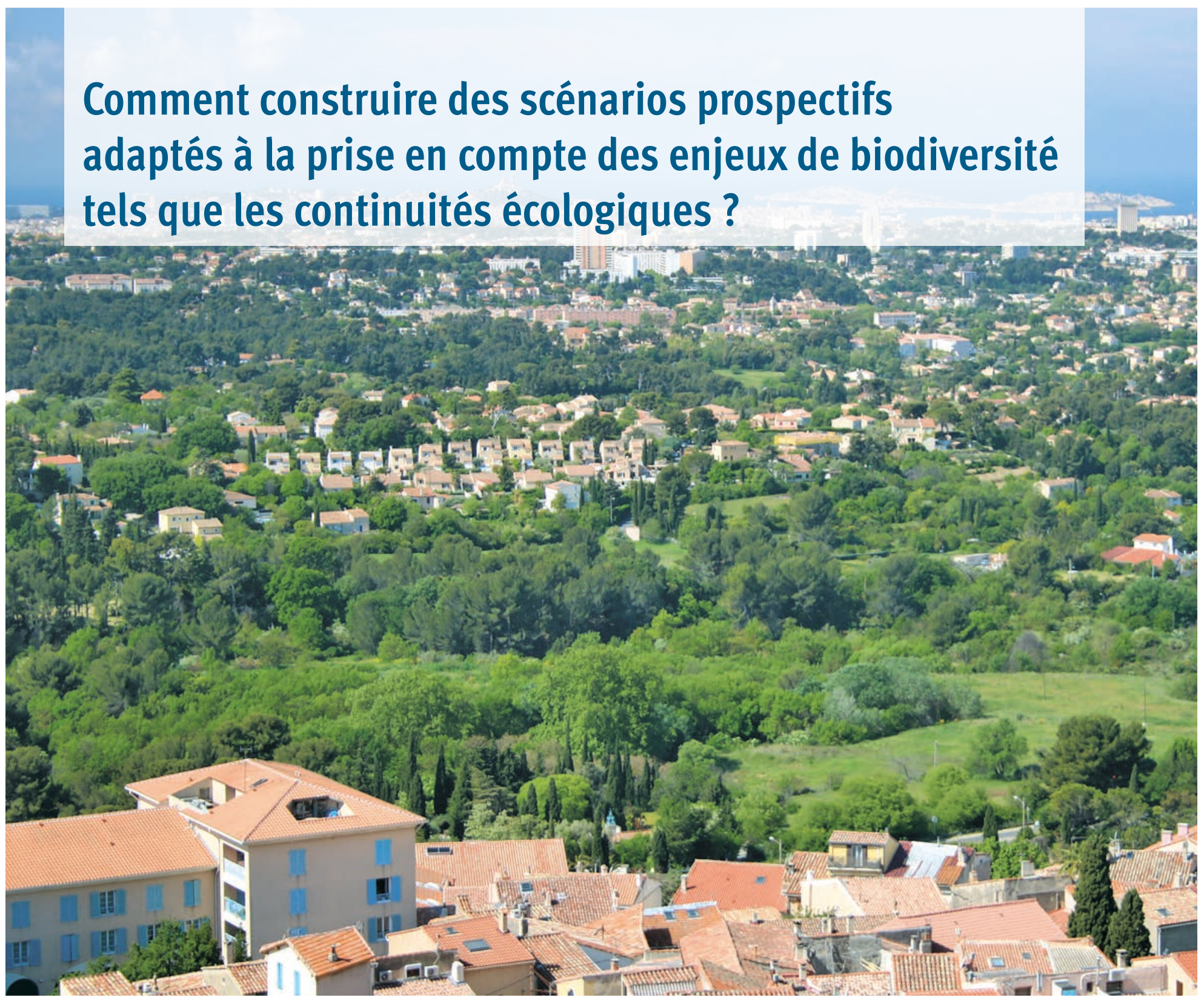




\title{
Comment construire des scénarios prospectifs adaptés à la prise en compte des enjeux de biodiversité tels que les continuités écologiques?
}

\author{
Pour préserver de manière efficace les continuités écologiques, il est essentiel de prendre \\ en compte l'évolution des territoires dans le temps et dans l'espace dans les stratégies \\ de conservation de la biodiversité. La démarche prospective, qui intègre les dynamiques \\ spatio-temporelles des territoires en impliquant les acteurs dans la construction de scénarios, \\ peut alors s'avérer un outil pertinent pour assurer le devenir à long terme de ces continuités \\ écologiques.
}

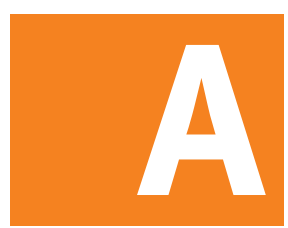

u regard des conclusions récentes de l'IPBES (the "International Platform for Biodiversity and Ecosystem Services »), force est de constater que conserver la biodiversité au travers de surfaces, même croissantes, d'aires protégées isolées ne suffit pas à endiguer son érosion et à préserver les services écosystémiques qu'elle procure à la société. Cela est particulièrement vrai dans un contexte de changements environnementaux rapides. De fait, I'amélioration des stratégies de conservation nécessite de préserver les dynamiques écologiques (ex.: flux de ressources et d'individus, capacité évolutive des espèces) qui permettent aux écosystèmes de s'adapter à ces changements. C'est ce que vise la mise en œuvre de la Trame verte et bleue (TVB), dans les Schémas régionaux de cohérence écologique (SRCE), intégrés désormais dans les Schémas régionaux d'aménagement, de développement durable et d'égalité des territoires (SRAD$\mathrm{DET})$. Ces schémas régionaux définissent les enjeux de « continuités écologiques » à l'échelle régionale, à travers I'identification des réservoirs de biodiversité (habitats de taille suffisante permettant la réalisation des cycles de vie de populations d'espèces) et leur mise en connexion avec des corridors écologiques. Ces enjeux sont également en cours de déclinaison à des niveaux territoriaux plus fins (métropole, intercommunalité, commune) dans les Schémas de cohérence territoriale (SCoT) et les Plans locaux d'urbanisme communaux ou intercommunaux (PLU(i)) notamment.
Cependant, l'efficacité de la conservation des continuités écologiques dépend de notre capacité à maintenir les potentialités dynamiques et les capacités adaptatives des écosystèmes; or les aménagements dans le territoire (création d'infrastructures de transports, replantation de haies...), les changements d'occupation ou d'usage du sol (urbanisation, abandon de parcelles agricoles - photo (1) et les dynamiques écologiques (ex.: enfrichement) influent sur l'efficacité de ces continuités écologiques. Ainsi, un espace d'habitat naturel peut jouer le rôle de réservoir de biodiversité ou de corridor écologique à un instant $t$ et se retrouver écologiquement isolé suite à des changements d'occupation et d'usage du sol dans le territoire à l'instant $t+1$. Pour assurer la préservation des continuités écologiques, il est donc essentiel de prendre en compte l'évolution des territoires dans le temps et dans I'espace - en plus des dynamiques des écosystèmes dans les stratégies de conservation. Cela nécessite d'être en capacité d'anticiper ces changements territoriaux et ce à différentes échelles d'espace et de temps.

Dans cette étude, nous proposons d'introduire la démarche prospective - c'est-à-dire l'exploration des futurs possibles pour éclairer l'action présente - et de montrer son intérêt pour améliorer la prise en compte efficace des continuités écologiques dans la planification territoriale. Au travers de scénarios et d'exercices participatifs (c'est-à-dire incluant des acteurs du territoire), ces démarches d'anticipation ont été appliquées de longue date pour l'aménagement du territoire (DATAR, 1971). 


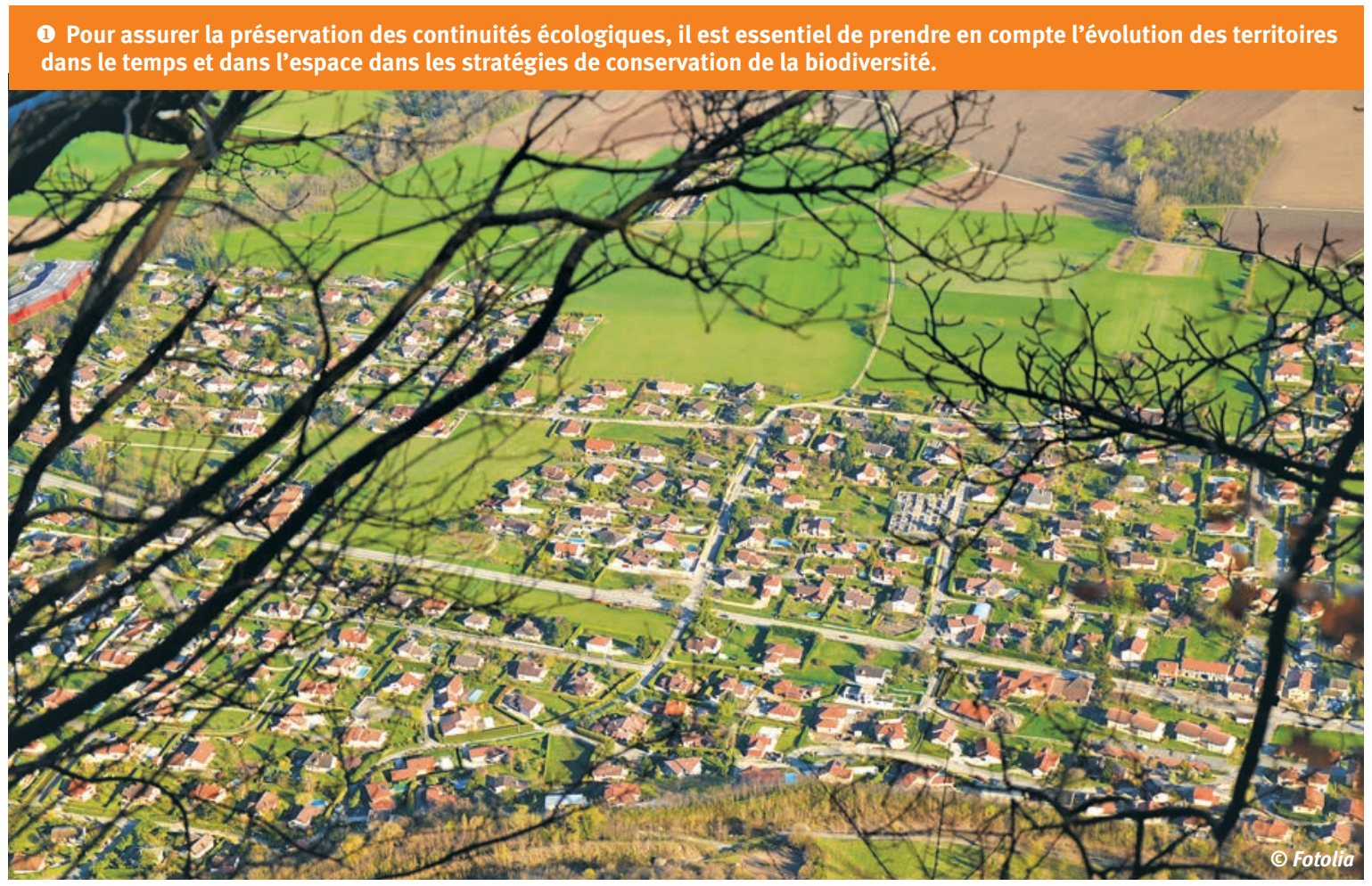

Récemment, la géoprospective a offert de replacer la dimension spatiale au cœur de la prospective en la combinant avec une modélisation fine des territoires et de leur devenir (Voiron-Canicio, 2012). La géoprospective pourrait ainsi se décliner en "écoprospective» qui, en appliquant la même démarche et les mêmes principes, permettrait d'explorer l'effet de scénarios d'évolution du territoire sur la biodiversité et d'en tirer les conséquences pour définir des futurs plus souhaitables en termes de conservation. Malgré une utilisation croissante de scénarios pour évaluer l'impact des changements globaux - surtout climatiques - sur la biodiversité, les changements d'occupation et d'usage du sol restent peu pris en compte pour les questions de biodiversité (Titeux et al., 2016), notamment à l'échelle territoriale. L'écoprospective permettrait de combler ce manque et pourrait s'appliquer, à l'instar de la géoprospective, en respectant les quatre phases principales suivantes :

- délimiter le territoire et en identifier les principaux facteurs de changements (démographie, facteurs socioéconomiques) sur la base des tendances passées et des enjeux associés à leur évolution future selon plusieurs horizons temporels $(2040,2080 \ldots)$;

- construire des récits, c'est-à-dire des histoires cohérentes d'évolution possible du territoire en faisant des hypothèses sur les tendances futures des facteurs de changements identifiés (ex.: augmentation des aides pour la conversion à l'agriculture biologique), éventuellement associées à des informations quantitatives et spatiales sur les changements ;

- retranscrire spatialement les récits à l'aide de la modélisation sous forme de cartes, appelées projections ou simulations spatiales. Ici, nous nous intéresserons en particulier au devenir du territoire selon des projections spatiales d'occupation du sol ;
- analyser les conséquences des possibles futures occupations du sol sur des enjeux spécifiques, comme ici les continuités écologiques, afin d'identifier des zones à enjeux de conservation ou d'évaluer différents scénarios de gestion du territoire.

Pour mener à bien une telle démarche "écoprospective» sur un territoire, on peut alors se demander quels scénarios de changements d'occupation et d'usage du sol devraient être utilisés ou construits. Dans cet article, nous présentons deux grands types de scénarios et leurs limites : les scénarios nationaux/supranationaux et les scénarios participatifs. Nous montrons ensuite comment les deux approches peuvent être associées dans le but de territorialiser les scénarios généralement utilisés aux échelles larges (ex.: Europe, niveau national) - c'est-àdire de les adapter à des contextes infranationaux (ex.: région, intercommunalité) en y intégrant des éléments locaux - par l'introduction de méthodes participatives généralement utilisées aux échelles fine (ex.: bassins versants). Dans un deuxième temps, nous discutons des apports des scénarios pour améliorer l'identification d'enjeux de conservation de biodiversité et de continuités écologiques.

\section{Quels scénarios pour anticiper les territoires de demain et à quelle échelle?}

De nombreuses typologies de scénarios existent dans la littérature (ex.: scénarios exploratoires qui analysent ce qu'il pourrait advenir vs. normatifs qui analysent ce que nous voulons faire). Nous avons également observé que les écologues travaillant sur des scénarios de biodiversité tendaient à utiliser des scénarios de changements d'occupation et d'usage du sol différents selon l'échelle à laquelle ils travaillent; par la suite, nous distinguons donc ces deux échelles. 


\section{Les scénarios nationaux ou supranationaux}

De nombreux scénarios socio-économiques ont été construits aux échelles nationales et internationales dans le but d'explorer les liens entre politiques internationales, tendances socio-économiques, et changements climatiques notamment. Ils décrivent l'évolution possible des conditions sociales, économiques, environnementales, démographiques et politiques (driving forces), et sont utilisés pour nourrir des modèles numériques complexes intégrant les dynamiques des cycles biogéochimiques, du climat et du fonctionnement sociétal (ex. : Integrated Assessment Models, IAMS). Un certain nombre d'éléments en découlent, dont les changements possibles $d^{\prime}$ occupation et d'usage du sol selon les différentes directions sociétales prises. Ces scénarios résultent des travaux de grands consortiums internationaux, comme par exemple le GIEC (Groupe d'experts intergouvernemental sur l'évolution du climat), ou de consortiums de projets de recherche européens (ex.: ALARM). Les exercices de prospective existant en Europe et en France explorent quatre grandes orientations de futurs possibles en croisant deux axes de changements : $1 /$ mondialisation vs. 2/ régionalisation de l'économie, et $\mathrm{A} /$ primauté à la consommation vs. B/ à la préservation de l'environnement. Ces scénarios ont pour atouts de proposer des évolutions quantitatives, souvent calibrées ou validées à partir de données passées et sont généralement associés à des projections spatialisées. Cependant, ils présentent aussi trois limites majeures qui les rendent difficilement utilisables pour explorer les changements d'un territoire ainsi que leurs conséquences sur la biodiversité. D'une part, ils ont souvent des résolutions spatiales et thématiques grossières $(>1 \mathrm{~km})$, même si cela est moins vrai avec les modèles plus récents qui ont des résolutions plus fines (ex. : 100 ou 250 m). Cela limite leur utilisation aux échelles infranationales et leur pertinence pour répondre à des questions de biodiversité qui nécessitent en particulier de s'intéresser à la configuration spatiale des paysages (Titeux et al., 2016). D'autre part, ces scénarios n'intègrent que rarement des spécificités régionales (ex. scénarios issus du programme ALARM, Spangenberg 2017), ce qui rend parfois les évolutions qu'ils proposent incohérentes avec les enjeux locaux. Par exemple, certains scénarios européens envisagent, dans le cadre du

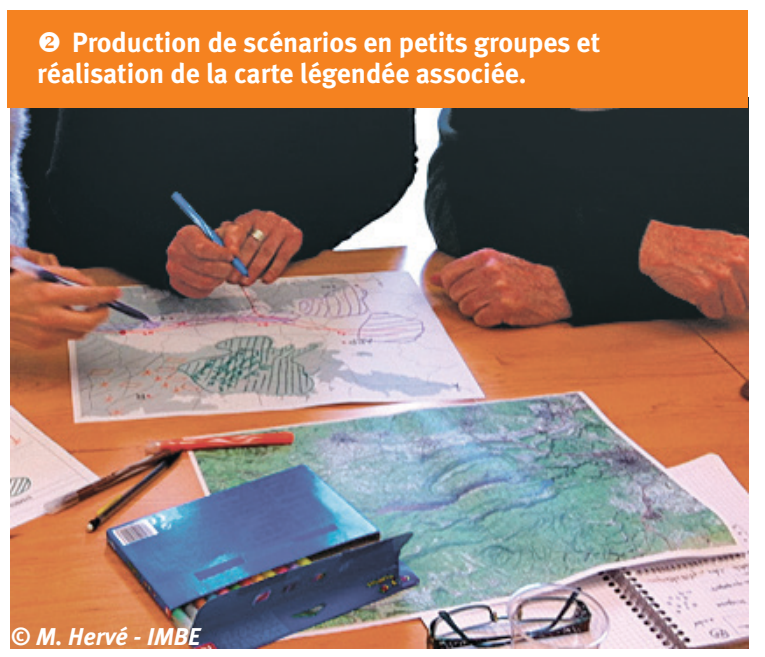

développement de la filière bois-énergie, la plantation de forêts sur les zones agricoles les plus productives de la région Provence-Alpes-Côte d'Azur. Une telle évolution semble peu probable, y compris pour un scénario rompant fortement avec les dynamiques actuelles, compte tenu de la demande croissante en production agricole locale. Enfin, il a été montré que, même lorsqu'ils reposent sur des récits très contrastés, les scénarios issus d'un même projet de recherche ou d'un même modèle ont tendance à proposer des évolutions très similaires, malgré des récits contrastés (Albert et al., 2020). Pour explorer une grande gamme de futurs possibles, il faudrait donc combiner des récits projetés issus de différents modèles. Or les incompatibilités thématiques (différentes classes d'usage du sol) et temporelles (différents horizons explorés) rendent cette option peu envisageable. Si cette démarche est préconisée dans l'étude des changements climatiques sur la biodiversité, elle n'a que très peu été appliquée à l'étude de l'effet des changements d'occupation et d'usage du sol sur la biodiversité (Albert et al., 2020).

\section{Les scénarios locaux}

De nombreux scénarios d'évolution des territoires ont également été construits par des groupes de travail impliquant les connaissances des acteurs locaux. Local ici est compris comme l'échelle d'un territoire subrégional présentant une certaine homogénéité : il peut s'agir d'une commune ou communauté de commune, mais également d'un bassin versant par exemple. Ces scénarios sont co-construits par une pluralité d'acteurs lors d'ateliers participatifs (photo (2), encadré (1)). La co-construction implique que la parole de chaque participant soit prise en compte (principe d'inclusivité) et soit considérée avec la même valeur (principe d'équivalence). Ces scénarios décrivant les tendances d'évolution possible d'un ensemble de facteurs de changements identifiés spécifiquement pour le territoire présentent trois atouts principaux. Tout d'abord, en s'appuyant sur les connaissances précises d'une diversité d'acteurs du territoire, ils intègrent des facteurs de changement pertinents pour ce territoire; ils permettent d'évaluer l'effet de décisions au niveau local. Ensuite, la connaissance des acteurs permet de caractériser l'occupation du sol de manière fine, en intégrant les usages (ex.: I'intensité de la production agricole ou de l'exploitation forestière) et une typologie de milieux adaptée aux enjeux du territoire. De plus, la coconstruction offre une meilleure compréhension (voire une appropriation) des enjeux et des processus décisionnels par l'ensemble des acteurs et des scientifiques impliqués (le scénario devient outil pédagogique). Les acteurs se trouvent également sensibilisés aux changements possibles dans leur territoire, à différents enjeux usuellement traités de manière sectorielle et à leur propre capacité d'action sur ces changements (Voiron-Canico, 2012).

Toutefois, ces scénarios présentent deux limites qui rendent difficile leur application aux questions de biodiversité. D'une part, les scénarios participatifs sont souvent produits sur des territoires spatialement restreints (bassin versant, commune) et finement cadrés sur les dynamiques et enjeux d'un territoire. Ils sont donc difficilement transférables aux territoires voisins. Or, I'anticipation des effets de l'aménagement du territoire sur la biodiversité doit être menée à des échelles multiples (du 
(1) Choix des acteurs. Ils doivent représenter une diversité d'enjeux du territoire et de points de vue (favorable/défavorable à une question, différents domaines d'expertise). La méthode « Factors, Actors, Sectors » permet de balayer les différents profils d'acteurs dans le territoire et d'identifier les individus pertinents pour chaque profil.

(2) Sensibiliser à l'intérêt de participer. La participation des acteurs étant bénévole, la présentation des objectifs et de l'intérêt d'une telle démarche est un élément déterminant pour mobiliser au mieux les acteurs.

3 Importance de l'animation. L'animation est un aspect clé du processus. L'animateur définit les règles d'échanges (bienveillance, écoute totale, équivalence de parole) qui permettent un déroulement fluide et constructif. Afin de réduire l'influence des jeux d'acteurs sur les résultats, il est en effet nécessaire de cadrer les comportements et attitudes des participants, en faisant respecter les temps de parole et en établissant une règle d'équivalence (la même importance est donnée aux paroles de chaque participant).

(4) Choix du format. Les différents formats (journée entière, demi-journées répétées, soirées) peuvent conduire à des résultats différents car ils ne mobilisent pas les mêmes acteurs (ex. : différence journée/soirée).

(5 La méthodologie de co-construction. Les acteurs peuvent être mobilisés pour leur expertise particulière ou au travers de jeux de rôle où chacun endosse le rôle d'un autre (ex. : un agriculteur « jouant » un élu). Les acteurs ne sont alors plus tenus à un discours dépendant de leur fonction et le déroulement de la co-construction s'affranchit des postures institutionnelles et corporatistes.

local, au régional, voire jusqu'au global), en particulier quand on focalise sur les enjeux liés aux continuités écologiques qui impliquent des dynamiques écologiques à différentes échelles spatiales et temporelles (Albert et Chaurand, 2018). Le cadre spatio-temporel de ces scénarios participatifs est donc difficilement compatible avec notre questionnement ou nécessiterait de répliquer I'exercice fastidieux des ateliers sur de nombreux territoires, avec des difficultés de coordination des différents exercices prospectifs par la suite. D'autre part, les tendances obtenues sont souvent qualitatives (ex. : description de l'évolution de l'urbanisation par mitage mais sans chiffrage précis). Ces exercices de prospective territoriale expriment les esquisses des changements futurs et doivent être couplés à une cartographie, voire à une modélisation spatialement explicite, pour les transformer en cartographies futures ou projections, comme proposé avec la géoprospective (Voiron-Canicio, 2012).

\section{Articuler tendances (supra-)nationales et spécificités locales afin de développer des scénarios aux échelles pertinentes pour la planification territoriale}

Les «échelles intermédiaires», entre le (supra-)national et le local (par exemple une région ou une interface entre régions) sont particulièrement pertinentes pour traiter des questions de planification pour la biodiversité, en particulier à travers la notion de continuités écologiques. En effet, celles-ci correspondent à un ensemble de niveaux biologiques spatialement imbriqués (du domaine vital de l'individu à la distribution géographique de l'espèce) qui transcendent les limites administratives et doivent être considérés conjointement pour maintenir les processus écologiques (Albert et Chaurand, 2018). Comme nous venons de le voir, les scénarios (supra-)nationaux et locaux présentent, malgré leurs atouts respectifs, des limites fortes à une utilisation directe aux échelles intermédiaires. Nous proposons donc de nous appuyer sur les scénarios (supra-)nationaux et sur les méthodes employées pour construire les scénarios locaux afin de produire des scénarios aux échelles intermédiaires intégrant à la fois des spécificités locales et des tendances fortes du (supra-)national.
On peut envisager trois grandes approches pour réaliser cette articulation, que nous nommerons scénarios «articulés».

Première option, les scénarios (supra-)nationaux sont territorialisés par des experts de la modélisation sur la base de données existantes. Les acteurs locaux peuvent alors être sollicités pour évaluer la pertinence de ces scénarios territorialisés et en souligner la vraisemblance ou les incohérences, au vu de leur connaissance du territoire. Dans une telle approche, les acteurs locaux ne sont impliqués que dans la phase finale du processus.

Deuxième option, les acteurs locaux sont impliqués dès le début du processus pour co-construire les scénarios territorialisés. Les scénarios (supra-)nationaux peuvent servir de lignes directrices pour fournir un contexte attendu au niveau (inter)national, cadre dans lequel les acteurs peuvent envisager les dynamiques de leur propre territoire (Houet et al., 2017). C'est la démarche utilisée dans le projet ESNET qui produit des scénarios au niveau de la "région grenobloise ${ }^{1}$ avec des acteurs locaux pour explorer les changements sur la biodiversité et les services écosystémiques (Bierry et Lavorel, 2016).

Troisième option, les scénarios (supra-)nationaux peuvent être utilisés en amont pour identifier les zones d'un territoire ou d'une région qui seraient les plus susceptibles aux changements, c'est-à-dire celles pour lesquelles un grand nombre de ces scénarios prévoient des changements à venir (tendance forte). On peut ensuite explorer les dynamiques de ces zones, en nombre restreint, grâce à des scénarios participatifs. Cette dernière approche permet le passage de cas locaux à la dynamique régionale, en se focalisant sur des cas contrastés et à forts enjeux pour une région.

Dans tous les cas, les récits de changements obtenus en articulant des éléments des scénarios (supra-)nationaux et des méthodes employées pour construire les scénarios locaux, peuvent ensuite être transcrits sous une forme spatialisée - cartes d'occupation et/ou d'usage du sol grâce à différents types de modélisation (Houet, 2015).

\footnotetext{
1. Correspondant à un niveau d'échelle intermédiaire.
} 
Apports des scénarios « articulés » comme aide à la décision pour faciliter l'identification d'enjeux pour la conservation de la biodiversité et des continuités écologiques

Le fait d'utiliser la prospective dans le cadre de questions d'aménagement du territoire en intégrant des enjeux de biodiversité ouvre un grand champ de possibilités. Les scénarios se présentent alors comme un outil d'aide à la décision permettant d'identifier des leviers de l'évolution du territoire et de proposer les actions nécessaires pour faciliter certaines trajectoires de changement. En effet, la construction de scénarios et de projections spatialisées associées permet de tester l'effet des changements tendanciels attendus dans le territoire (ex.: expansion urbaine, nouvelle infrastructure) sur les enjeux de biodiversité, telles que les continuités écologiques (Albert et al., 2017). Comparer les valeurs (ou cartes) futures des indicateurs de biodiversité avec les valeurs (ou cartes) actuelles peut alors donner des arguments pour réviser les politiques publiques ou prioriser les enjeux de conservation. C'est par exemple ce qui a été fait dans le projet de recherche ESNET, dans lequel I'exploration des dynamiques territoriales futures a permis d'en analyser les conséquences possibles sur les services rendus par les écosystèmes dans le territoire (Biery et Lavorel, 2016).

Quatre grands types de contributions sont envisageables.

\section{Initier des changements au sein d'un territoire}

Travailler sur des scénarios permet de déterminer l'adaptabilité d'un territoire en identifiant les facteurs de changement possibles puis en quantifiant la marge de manœuvre ou les effets de seuils spécifiques au territoire pour ces facteurs (Voiron-Canicio, 2012). Les scénarios n'offrent pas seulement une anticipation des tendances et une représentation de l'avenir, ils permettent également de modeler le futur et de définir un cheminement pour orienter l'action publique (Musso, 2012).

\section{Tester l'effet de projets}

Les scénarios et projections spatialisées sont des moyens de tester en amont l'impact potentiel d'un aménagement et de ses alternatives, en alimentant notamment les réflexions de la séquence "Éviter, Réduire, Compenser » à mettre en œuvre pour les projets d'aménagements. $C^{\prime}$ est ce qui a été testé dans le projet ITTECOP E=RC+ (Tatoni et al., 2020) ${ }^{2}$. Cela permet d'identifier la localisation optimale d'un projet, en réalisant un compromis entre l'impact écologique et les contraintes de réalisation du projet pour répondre au volet «Éviter» de cette séquence. Les scénarios permettent aussi d'optimiser les mesures compensatoires en fonction de l'évaluation de leur efficacité. Ils peuvent également tenir compte de l'effet cumulé d'un ensemble de projets susceptibles de modifier les impacts écologiques.

\section{Prioriser les enjeux de conservation \\ face à l'enjeu des changements globaux}

Les scénarios sont potentiellement un outil de priorisation des enjeux de conservation au regard des changements à venir, en focalisant plus particulièrement sur i) des zones à enjeux écologiques à la fois dans le présent et dans le futur ou des zones à enjeux écologiques futurs, ou ii) des zones ayant des enjeux écologiques actuels forts et de fortes pressions dans le futur (hot-spot de changement) afin de les prioriser ou d'orienter les choix de conservation vers des zones sous moindre tension. Ils permettent également de tester l'efficacité d'un plan de conservation (création d'aires protégées), basé sur des critères actuels (continuités écologiques) et de tester sa robustesse dans un contexte de changements à venir.

\section{Communiquer plus facilement sur une vision intégrée du développement du territoire}

Les scénarios sont enfin un bon support pour communiquer sur les aménagements et leurs conséquences car ils illustrent les effets de décisions sur le territoire. Ils peuvent également servir de supports à un partage d'enjeux afin d'élaborer avec les acteurs locaux un projet de territoire ou de décider de plans d'actions, permettant ainsi de susciter le dialogue, confronter les acteurs aux conséquences de certains choix et définir des stratégies (Voiron-Canicio, 2012). Différentes thématiques, autres que la biodiversité, peuvent compléter l'approche pour donner une vision plus intégrative du territoire (ex.: transport, économie, dimension sociétale, etc.), tout en précisant toujours les limites des scénarios utilisés et les incertitudes qui y sont associées.

\section{Conclusion}

La géoprospective s'est développée depuis quelques années en géographie pour répondre au double enjeu d'anticiper le futur et d'en spatialiser les effets. Utilisée dans le cadre de la mise en œuvre de la TVB et de la conservation de la biodiversité, cette posture permettrait de proposer des éléments d'analyse pour mettre en œuvre un aménagement du territoire plus cohérent car tenant compte $d^{\prime}$ un ensemble de changements (projets à venir, impacts cumulés...), plus fiable car anticipatif des changements futurs (changement d'occupation et d'usage du sol, changement climatique) et plus intégratif des différents enjeux (sociaux, économiques et écologiques).

Pour utiliser cette approche à des échelles intermédiaires, il semble important d'intégrer différentes sources d'informations sur les évolutions possibles et souhaitables des territoires. S'appuyer sur les apports respectifs des scénarios (supra-)nationaux et de scénarios participatifs semble une solution intéressante pour allier les avantages et remédier aux limites de chacun de ces deux types de scénarios. L'un et l'autre peuvent en effet s'enrichir pour proposer à la fois des tendances locales et globales interagissant au niveau territorial. Or des échelles territoriales multiples sont nécessaires pour évaluer le devenir et la préservation des continuités écologiques. Anticiper et construire les dynamiques territoriales futures est également nécessaire pour une meilleure application de la séquence «Éviter, Réduire, Compenser » dans le cadre de projets d'aménagement. 


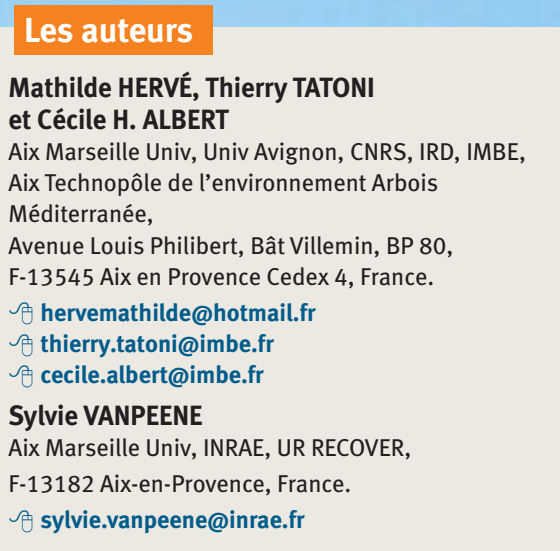

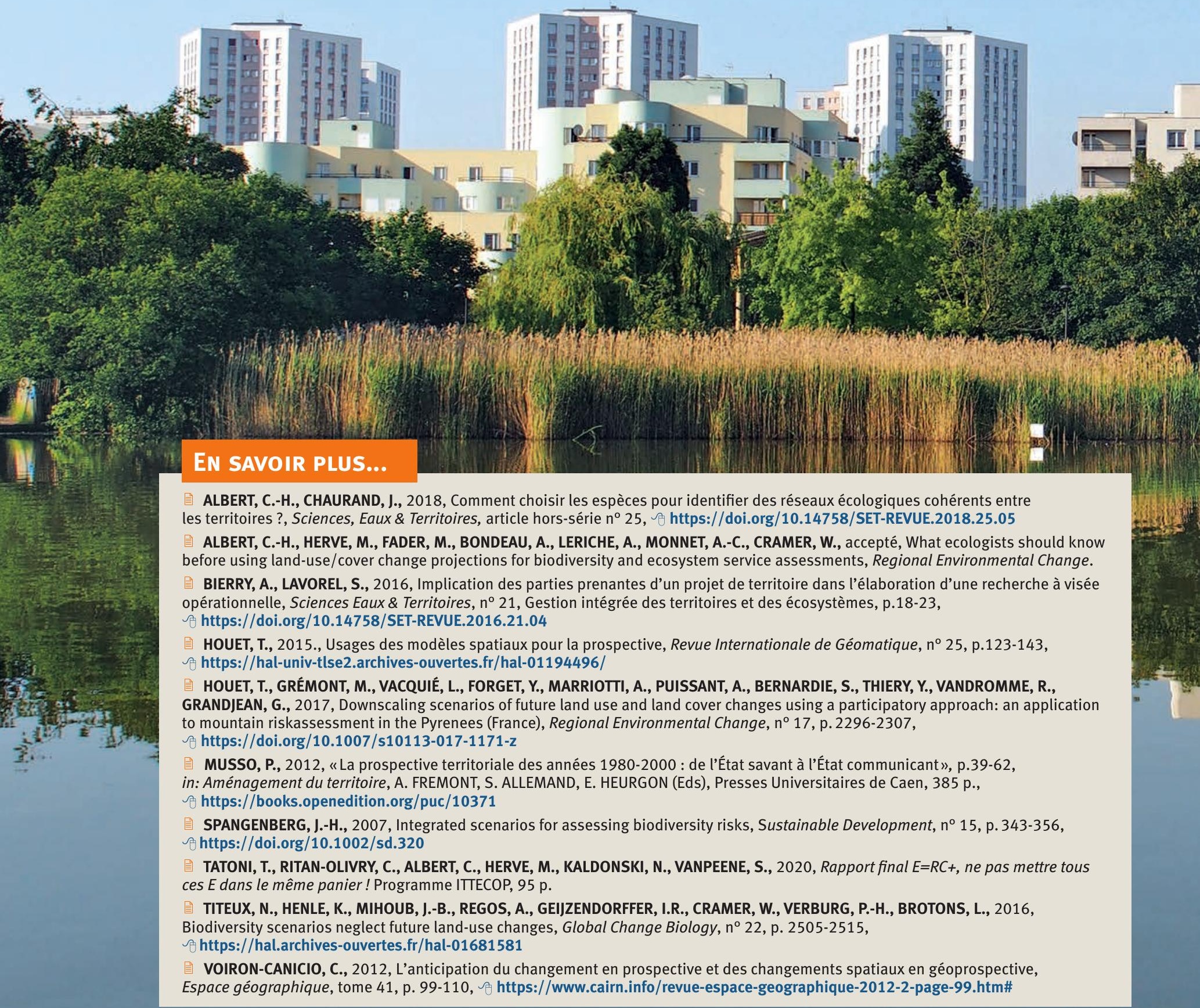

